\title{
UAV based wilt detection system via convolutional neural networks
}

L. Minh Dang ${ }^{1}$, Ibrahim Hassan Syed ${ }^{1}$, I Suhyeon ${ }^{1}$, Arunkumar Sangaiah ${ }^{2}$, Irfan Mehmood ${ }^{1}$, Seungmin $\mathrm{Rho}^{3}$, Sanghyun $\mathrm{Seo}^{3}$, Hyeonjoon Moon ${ }^{1^{*}}$

${ }^{1}$ Department of Computer Science and Engineering, Sejong University, Seoul, Republic of Korea;

${ }^{2}$ School of Computing Science and Engineering, VIT University, Vellore, India

${ }^{3}$ Department of Media Software, Sungkyul University, Anyang, South Korea

*Correspondence: $\underline{\text { hmoon@sejong.ac.kr }}$

\begin{abstract}
The significant role of plants can be observed through the dependency of animals and humans on them. Oxygen, materials, food and the beauty of the world are contributed by plants. Climate change, the decrease in pollinators, and plant diseases are causing significant decline in both quality and quantity of the plants and crops on global scale. In developed countries, above 80 percent of rural production is produced by sharecropping. However, due to widespread diseases in plants, yields are reported to have declined by more than a half. These diseases are identified and diagnosed by the agricultural and forestry department. Manual inspection on a large area of fields requires a huge amount of time and effort, thereby reduces the effectiveness significantly. To counter this problem, we propose an automatic disease detection and classification method in radish fields by using camera attached on Unmanned Aerial Vehicle (UAV) to capture high quality images of the fields and analyze them by extracting both color and texture features, then we perform segmentation on them using Kmeans clustering to select radish regions in the field and feeds them into convolutional neural network $(\mathrm{CNN})$ to detect Fusarium wilt of radish efficiently at early stage and allow the authorities to take timely action which ensures the food safety for current and future generations.
\end{abstract}

Keywords. Unmanned aerial vehicles, radish field database, radish field segmentation, Fusarium wilt of radish classification

\section{Introduction}

In Korea, radish is considered the national vegetable, occupying about $10 \%$ of the entire vegetable farming area. It is an indispensable ingredient in soups, stews, and other dishes. However, the yield of radish has decreased sharply because Fusarium wilt of radish has emerged at an unprecedented rate. The symptoms include wilting, chlorosis, necrosis, premature leaf death and a deterioration of the vascular elements in roots, stems, and petioles which lead to imminent death of the infected plant [1]. It is challenging to prevent and treat the 
disease due to several reasons. When the disease appears, it spreads rapidly from infected plants to healthy plants, resulting in severe harvest losses. Early detection of the disease could prevent the widespread of the disease and mitigate the damage. Manual field inspection has been applied for a long time, but it is inefficient, and time-consuming. Therefore, an automated, fast, and precise surveillance system for detecting Fusarium wilt of radish will likely become reality more than ever as advanced technologies such as UAVs, IoT application, and remote sensing are emerging.

Remote sensing is part of a GIS system which supports the measurements of agricultural area. Satellite and airplanes have been two major remote sensing technologies for a long time. The results acquired from satellite remote sensing are affected by poor resolution image or inaccurate information due to poor weather conditions and high costs [2] whereas in airplanes remote sensing, the plane is equipped with multiple sensors and cameras which provide high quality and detailed information, yet the equipment is expensive and hard to operate [3]. As an alternate method, unmanned aerial vehicle (UAV) is a remote-controlled aircraft which records the interested surface at relatively low-altitudes [4]. Owing to the advances in sensing technology, control, and positioning techniques, UAV is now capable of acquiring high spatial resolution surface images at low operational cost. Because it has multiple functions as well as its cost-effectiveness, the applications of UAV are rising rapidly. It can be applied in many areas from traffic monitoring [5] to forest fire monitoring [6] and search \& rescue operations [7]. In addition, UAV has a promising potential in converting precision farming into autonomous farming [8]. It assists farmers in observing crop and field information in a timely manner which lead to the improvement in crop management and farm planning.

Beside remote sensing, Internet of things (IoT) concepts is emerging and has drawn much attention to the research community recently [21,27,28,29]. IoT lets various devices around us communicate and collaborate with each other. In case of radish farming, multiple sensors have already been placed on the field to collect periodic scalar data such as light, temperature, humidity, etc. then the data are sent directly to the main controller for analyzing and automatically adjusting the optimal environmental parameters for radish. As the UAV applications have rocketed in recent years, it can be integrated to existing IoT system on the field. By letting the drone flies over pre-programmed locations at specific time on daily basis to record multimedia contents such as video and images, these contents are then sent to the main controller for detecting the wilt of radish in real-time and notifying the users detailed information by sending message if the disease is detected. 
Traditional approaches in image representation relied heavily on extracting handengineered features like color, texture and shape features $[9,10]$, scale invariant features transform (SIFT) [11], and bag-of-visual words [12]. Those systems relied on shallow classification methods like support vector machines [22], decision tree [24], random forest, dependencies of inter-block coefficients [30], multi-scale non-negative sparse coding [31], multi-factor feature [32, 33], swarm intelligence [25] and neural networks for identifying plant diseases [26]. However, the low discriminative ability of these features and their failure to describe high level semantics resulted in poor classification performance. Recent successes in deep learning based methods in image classification tasks have received much attentions of computer vision researchers to utilize these powerful hierarchical architectures for various tasks [13]. While modern CNN architectures do require a huge computational power, efforts have been made to reduce their computational needs while keeping their performance as high as possible. Consequently, several efficient $\mathrm{CNN}$ architectures have been proposed like GoogleNet [14], Network-in-Network [15].

Based on the above analysis and considerations, we proposed a novel approach to combine UAVs with computerized methods for detecting the criticalness of Fusarium wilt of radish. The main contributions include four aspects:

- Detecting and categorizing the criticalness of Fusarium wilt of radish.

- Segmenting the radish regions from other regions on the field such as bare ground and mulching film.

- Classifying the severity of the Fusarium wilt of radish based on thresholding a range of color features.

- Creating two different datasets. One dataset contains high resolution radish field images obtained from the drone. The other dataset contains images for radish, bare ground and mulching film regions which were manually verified and collected. They will be made public for the research community for further experimentation and simulations.

\section{Datasets}

\subsection{Image Acquisition}

The images used in this study were captured in different areas in Korea between July and September 2016. Two commercial UAVs (Phantom 4, DJI co., Ltd.), equipped with RGB camera (12 mega pixels), were used to obtain the images at the altitudes of approximately $10 \mathrm{~m}$ 
above the ground level. A total 40 images were acquired and each image has dimensions of $4000 \times 3000$ pixels.

\subsection{Dataset Preparation}

From the original 40 images, two datasets were created. Dataset A contains manually cropped and labeled regions of interest (ROIs) representing three regions: radish, bare ground, and mulching film as shown in Fig. 1. In total, 1,500 ROIs were extracted; 500 ROIs for radish, 500 ROIs for bare ground, and 500 ROIs for mulching film regions. Dataset A is used for radish region classification. Dataset B contains the original 40 images of size (4000x3000) pixel.

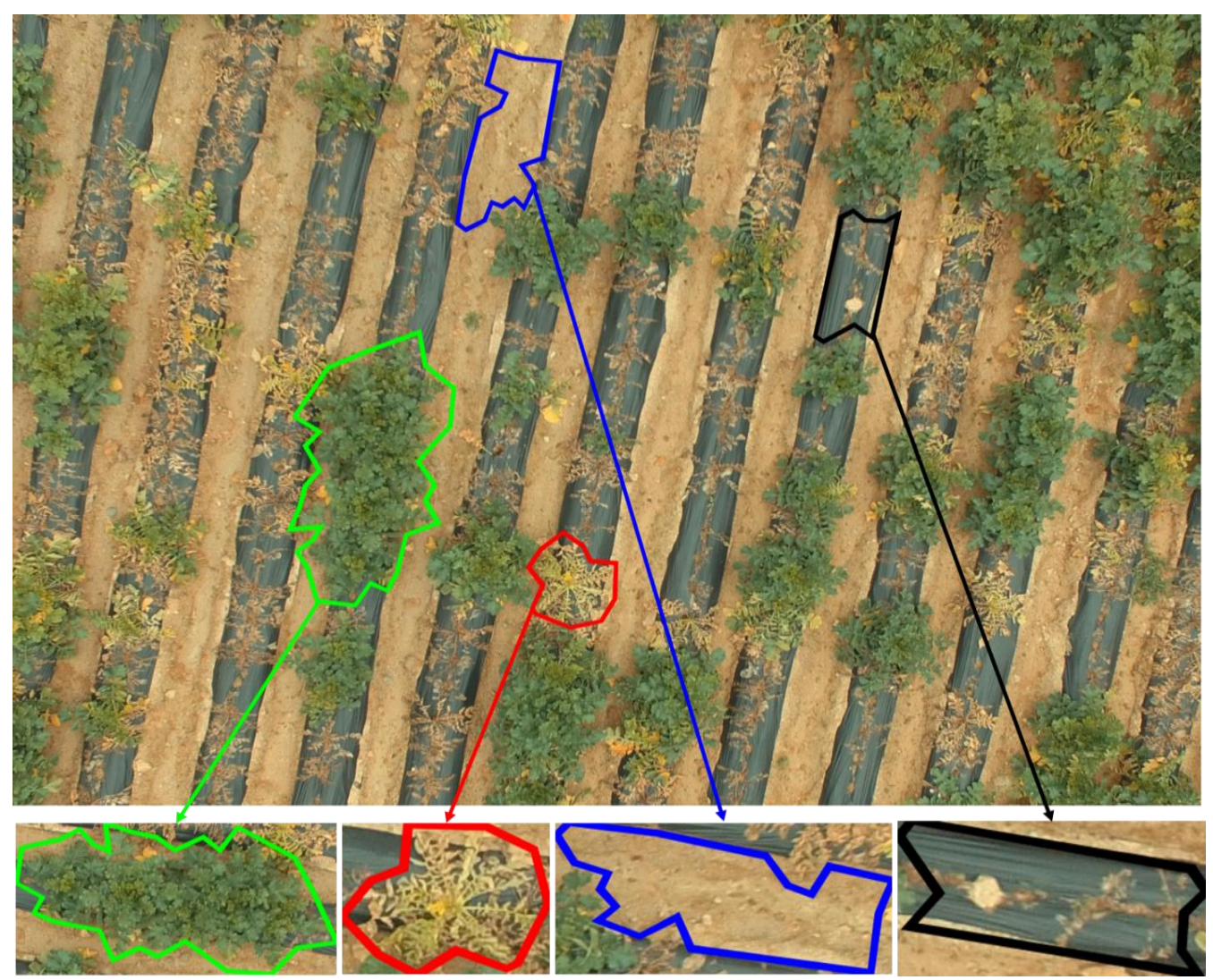

Figure 1. Sample image of radish field. Colored regions indicate different regions of radish fields (Green: Healthy radish, Red: Fusarium wilt of radish, Blue: Bare ground, Black: Mulching film)

\section{Methodology}

As shown in Fig. 1, the whole radish field contains three main regions (Radish, bare ground, and mulching film). The final goal of this study is to detect Fusarium wilt on radish leaves so segmentation is needed to divide the radish field in Dataset B into distinct regions. Next, a classifier is trained to recognize which region is radish using Dataset $\mathrm{A}$, the output after applying this classifier to the segmented regions is radish regions. After that, by sliding a fixed 
size rectangular window $(64 \times 64,128 \times 128,256 \times 256)$ pixel through the radish regions from the segmentation step, a list of small size radish images was extracted, each image was assigned a label indicates level of disease by applying various image processing methods. Finally, a convolutional neural network $(\mathrm{CNN})$ model is implemented for classifying Fusarium wilt of radish based on the severity of the disease. The overall process of the proposed model is shown in Fig. 2.

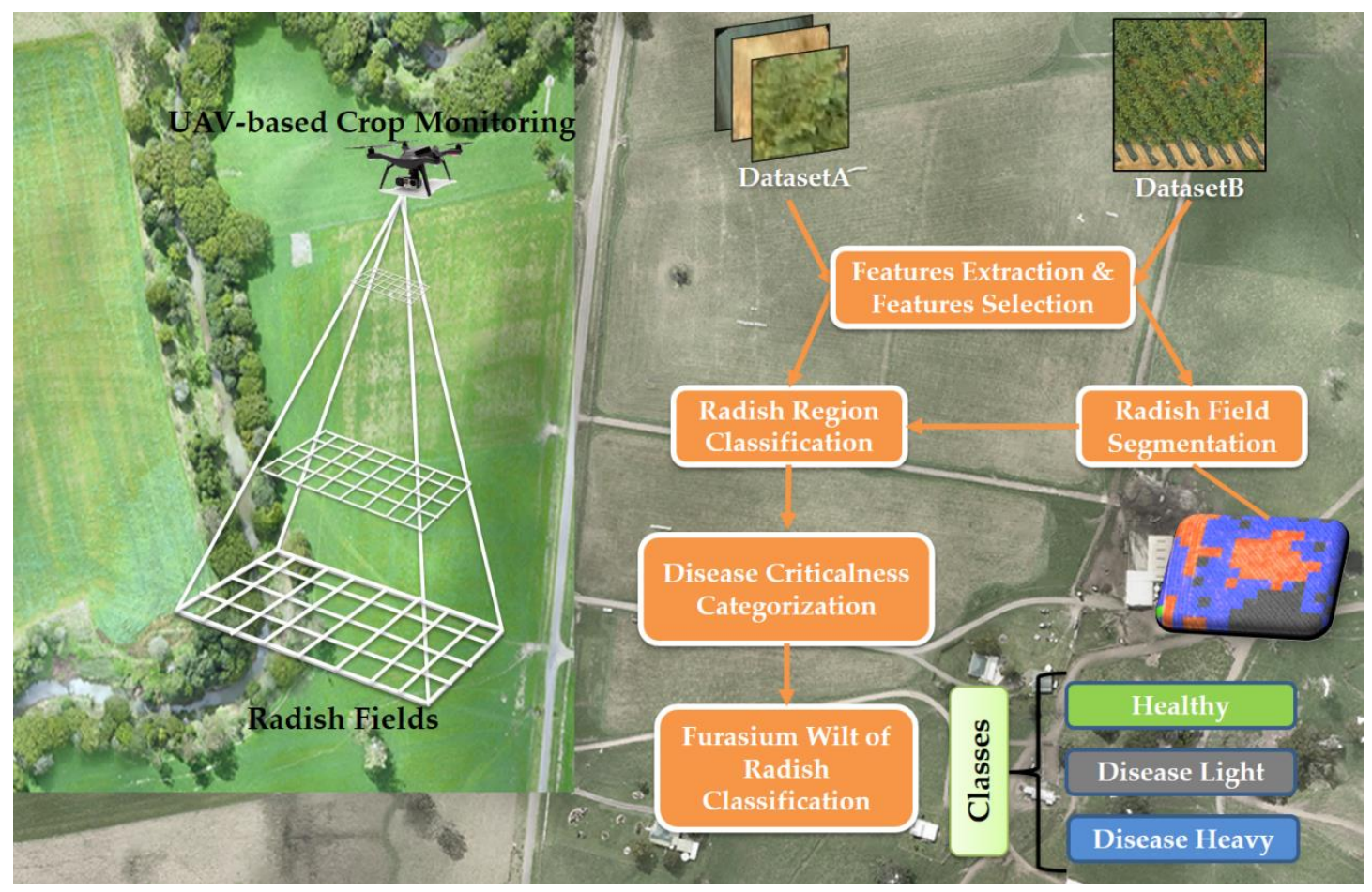

Figure 2. Overview of the proposed method for Fusarium wilt of radish classification

\subsection{Features Extraction \& Features Selection}

An image contains various useful features such as: color, shape, texture. Each type of feature serves specific purpose in image processing. However, image information retrieval using only one kind of feature is not good enough for the accuracy and efficiency of the model. High dimensional feature lower the model efficiency whereas low dimensional feature decrease model accuracy, it is better to use multi features for feature retrieval. Since, color and texture are the most important visual features, they are extracted in this study. Local binary pattern (LBP) in [16] was applied to extract texture features from radish image whereas color features were extracted by using color-space conversion. After that, two featured sets were combined into one feature vector then AutoEncoder (AE) [17] was utilized to reduce the feature dimension. 


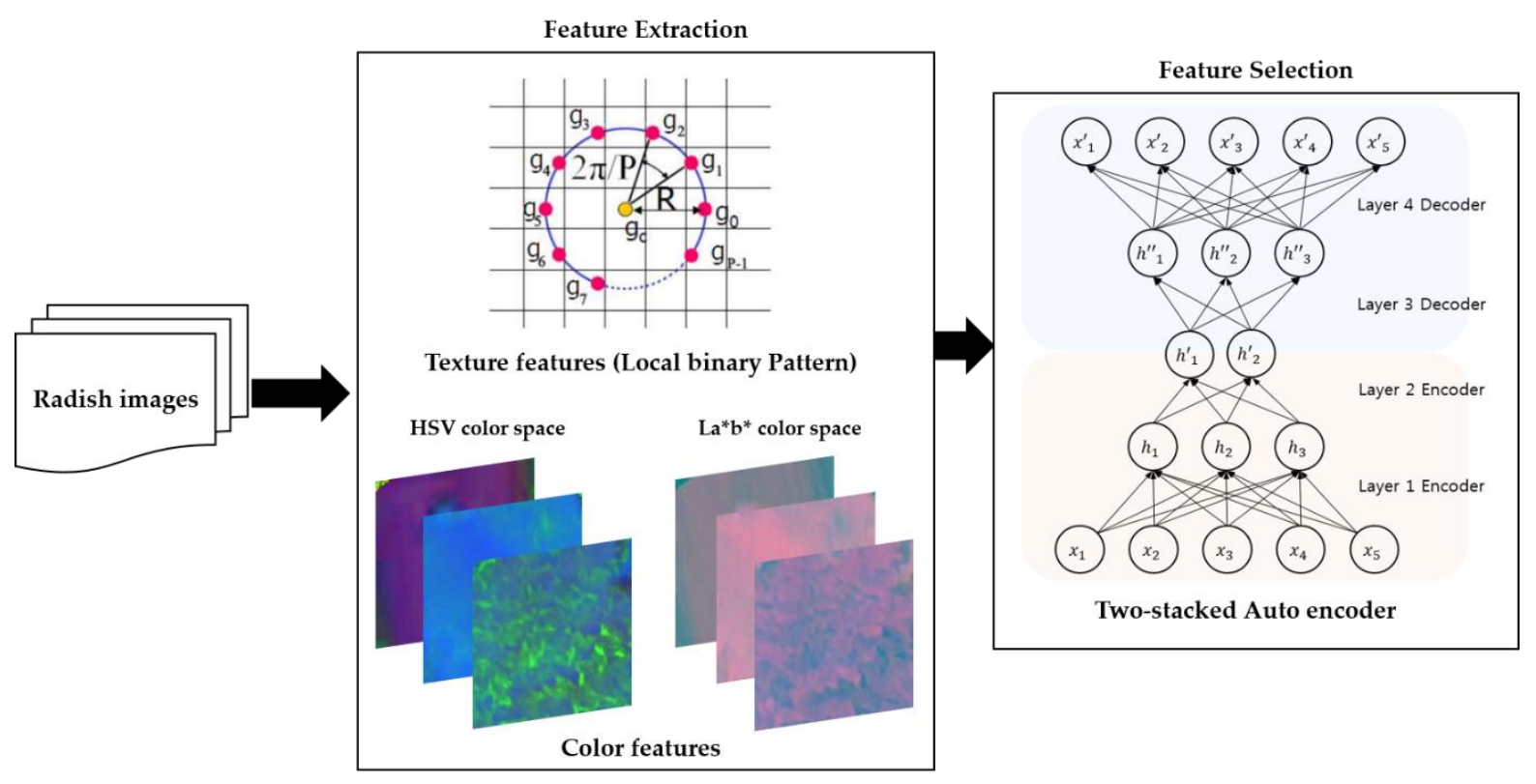

Figure 3. Features extraction and features selection process

\subsubsection{Features extraction}

Local binary pattern provides a robust texture descriptor which is invariant to rotation and illumination changes at low computational cost. Input a (center) pixel $\mathrm{c}$ in an image, LBP takes its neighboring pixels (a set of regularly distributed pixels on a circle) $p(p=0, \ldots, P-1)$ in a radius $\mathrm{R}$ and generates a binary pattern vector as shown in below formula

$$
L B P_{P, R}=\sum_{p=0}^{P-1} s\left(g_{p}-g_{c}\right) 2^{p}
$$

where $\mathrm{s}(\mathrm{x})$ is 1 if $\mathrm{x} \geq 0$ or 0 if $\mathrm{x}<0, g_{c}$ and $g_{p}$ indicate the grayscale of the center pixel and its neighborhood pixels, respectively. However, the binary pattern vector generated above is still sensitive to rotational invariance, so Equation (2) is a solution to overcome this problem.

$$
L B P_{P, R}^{r i}=\min \left\{R O R\left(L B P_{P, R}, i\right) \mid i=0,1, \ldots, P-1\right\}
$$

where $\operatorname{ROR}(x, i)$ is computed by a circular bitwise right shift operation which means that the same binary pattern code generated by the bitwise operation is regarded as one identical pattern. In our case, LBP features are computed on a gray-scale image using three neighboring topologies $(\mathrm{P}, \mathrm{R})=\{(8,1),(16,2),(24,3)\}$ which generated 703,404 features.

Radish field images were captured in RGB (red, green and blue) color space then converted into HSV (hue, saturation, value) and L*a*b (lightness, green-red, blue-yellow) color space for a better processing result. Then the histograms were built on hue, *a, and *b channels (256 bins or features per histogram). After that, three color histograms were concatenated into one color histogram, generating approximately 768 color features 


\subsubsection{Feature selection}

After extracting texture features and color features, they were combined into one single features vector. However, two features cannot be combined directly because there is a huge difference between their dimension, therefore the classification and segmentation step will be influenced by the texture features which had much more dimensions than the color features. This imbalance will probably affect the accuracy of the model. The solution for this problem is to scale the dimensions of these two features to the same dimensions. Initially, texture features $t_{i}$ is shrunk whereas color features $c_{i}$ is extended so each features set will occupy half of the dimensions $d_{x}$ of the input vector $x_{i}$.

The input vector $x_{i}$ is made by concatenating texture features $T_{i}$ and color features $C_{i}$, which are the shrunken texture features of $t_{i}$ and extended color features of $c_{i}$, respectively. $T_{i}$, $C_{i}$ and $x_{i}$ are computed as follows:

$$
\begin{aligned}
T_{i} & =W_{t} t_{i}+b_{t} \\
C_{i} & =W_{c} c_{i}+b_{c} \\
x_{i} & =\left[T_{i} C_{i}\right]
\end{aligned}
$$

where the matrices $W_{t} \in R^{d_{x} \times d_{t}}$ and $W_{c} \in R^{d_{x} \times d_{c}}$ represent the weights, $b_{t}$ and $b_{c}$ represent their biases.

After applying scaling, the texture features $t_{i}$ was reduced from 703,404 to $T_{i}=10,000$, and color features $c_{i}$ was extended from 768 to $C_{i}=10,000$. As the result, the input vector $x_{i}$ contains a total of 20,000 features. The number of features were in very high dimensional space so a features selection method is implemented to improve the system performance. AutoEncoder (AE) [17] is an unsupervised learning technique, typically used for dimensionality reduction. It includes the input and output layer (of the same dimensionality) and hidden layer(s). It tries to learn an approximation/representation of the input. The dimensionality of the hidden layers is smaller than the input and output layers. The hidden layers learn the compressed representation of the input (encoding), i.e., extracting meaningful features from the input. Finally, the input features were reduced and compressed by applying two-stacked AE [18] on 20,000 features to only 1,770 features.

\subsection{Radish Region Classification}

A softmax classifier is constructed for radish field classification, it computes the probability that a region belongs to a specific class. The class which has the highest probability is assigned to the region. It is a generalization of logistic function that can be used for multi-class 
classification. It is mainly deployed as a final classification layer for neural network based model. Given a feature vector $x$, the softmax classifier outputs the probability for each class label $j(j=1, \ldots, C)$ as follows:

$$
\begin{aligned}
& P(y=j \mid x)=\frac{e^{u(x) j}}{\sum_{k=1}^{K} e^{u(x) k}} \quad, \text { for } j=1 \ldots . C \\
& u(x)_{j}=\sum_{i=1}^{I} w_{i j} \cdot x_{i}+b_{j}
\end{aligned}
$$

In which $y_{a}$ represents the class label, $w_{i j}$ is the weight and $b_{j}$ is a bias $(i=1, \ldots, I, j=$ $1, \ldots, C) . I$ and $C$ denote the number of features and classes (radish, bare ground, and mulching film), respectively. The weight $\mathrm{w}$ and bias $\mathrm{b}$ are computed to minimize mean squared error (MSE) with 200 iterations. Equation (6) is called softmax function that return a C-dimensional vector of real values between 0 and 1 , representing the categorical probability distribution. The class which has the highest probability is assigned to $x$.

Support vector machine (SVM) was also implemented to compare with softmax classifier to find the optimal classifier for the dataset, SVM is proved to be one of the most efficient algorithms for classification problem [22]. It uses decision boundary to separate sample from different classes. The SVM kernel used in this study is linear kernel because the number of features were larger than the number of training samples so there is no need to map data to a higher dimensional space and we only needed to search for the suitable parameter C. LibSVM [23] is a library for implementing support vector machines (SVM). It helps the users implement and customize SVM easily to fit their case. After using cross-validation supported by the library, the best values for $\mathrm{C}$ was 0.5 .

\subsection{Radish Field Segmentation}

In the entire radish field image, radish is the only region of interest for further steps, thus $\mathrm{K}$-means clustering is implemented to segment the radish field image into distinctive regions and only the radish regions are collected. Fig. 4 shows the entire process of radish field segmentation. Features are extracted from original radish RGB field image. Then, K-means clustering is performed with $\mathrm{K}=3,5,10,15$ and 20. For each cluster, the radish field classifier from previous section is used to assign a class label (radish, bare ground and mulching film) to each cluster.

After filtering out the radish regions through K-means clustering, a fixed window was created to slide through the region, and the window size was set to 64, 128, and 256 sequentially so we can figure out which window size achieves the best Fusarium wilt of radish classification results. 


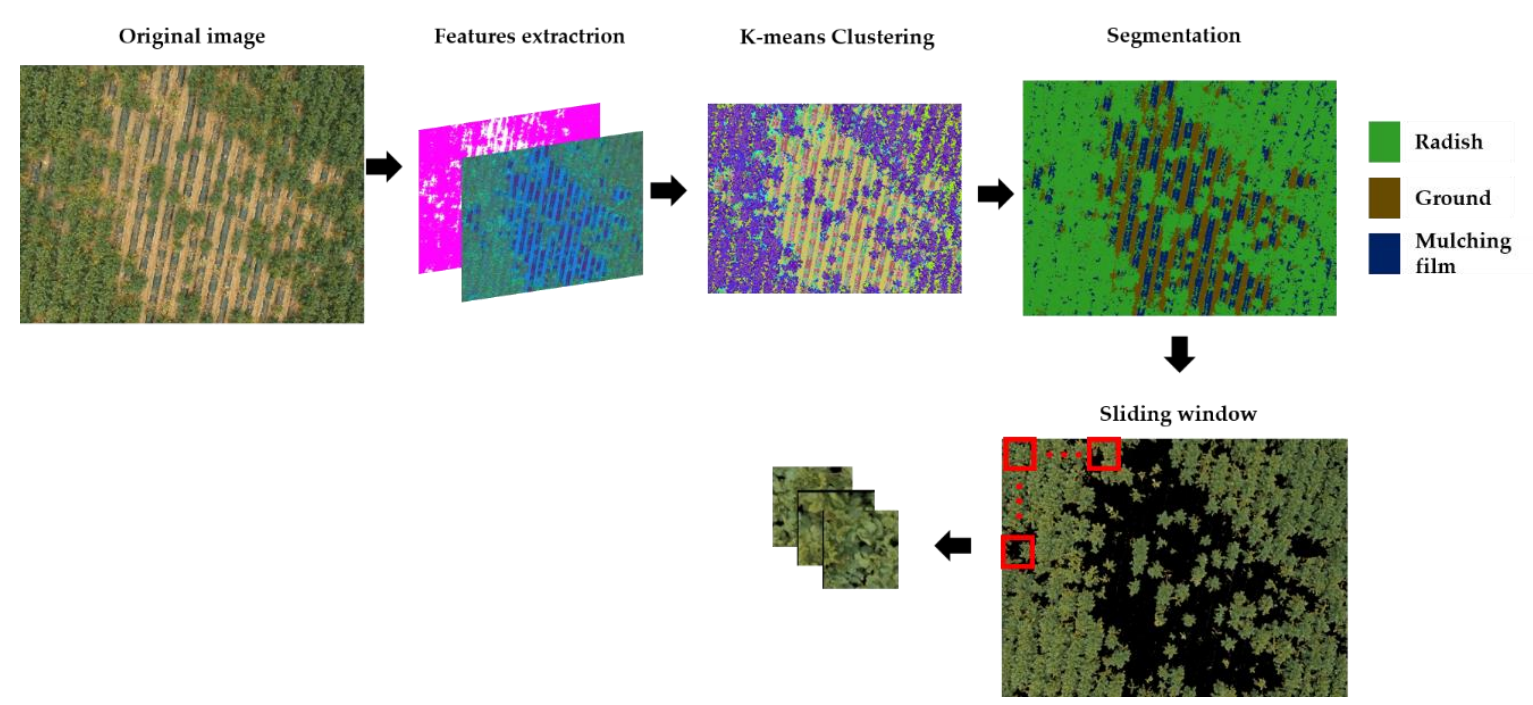

Figure 4. Overview of radish field segmentation process

\subsection{Disease Criticalness Categorization}

As the criticalness of Fusarium wilt appears in the radish at many stages, farmers have specific treatment plan for each stage. Early and accurate detection and diagnosis of plant diseases is the key factor in plant production and the reduction of both qualitative and quantitative losses in crop yield. Fig. 5 shows sample ROIs for healthy, light and heavy disease.

Each image extracted from the segmentation step was converted from RGB to HSV color space, and after that, thresholding operations were performed by applying a range of pixel values representing the greenish region. When applying the thresholding technique, the portion of black pixel represents yellowish region (Fusarium wilt) while the portion of white pixel represents the greenish region (healthy radish). Radish that suffered from more severe disease have more black pixels in the threshold image than healthy radish or radish suffered from early stage of the disease. Therefore, the following properties are used to set a threshold to classify each input image into one of these three categories: healthy, light disease, and heavy disease as illustrated in Fig. 5. If the percentage of white pixels in the image is over or equal to $90 \%$, it is categorized as healthy radish. For the disease light radish, it is less than $85 \%$ and over $65 \%$. The image is categorized as disease heavy radish if the percentage of white pixels is less than $65 \%$. As the result, for each window size $(64,128,256), 6,000$ images were extracted including 2,000 for healthy radish, 2,000 for light disease and the other 2,000 images for heavy disease 


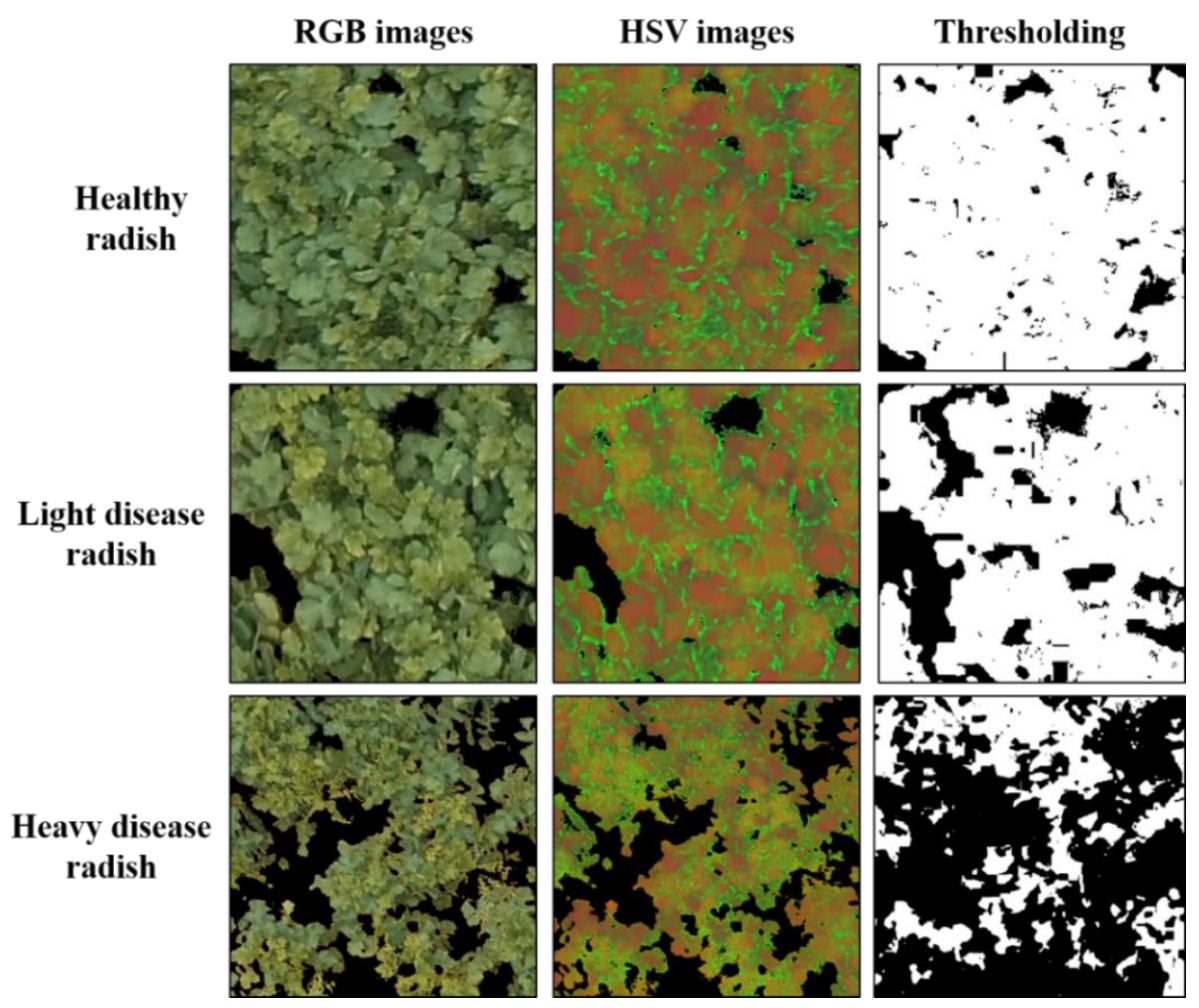

Figure 5. Sample criticalness categorization on (128x128) pixel window size

\subsection{Fusarium Wilt of Radish Classification}

In order to detect and classify the criticalness of the Fusarium wilt of radish, a well-known CNN model GoogleNet [14] is adopted. This CNN model was proposed in the ImageNet large scale visual recognition challenge [19], this CNN model achieved a remarkable performance of 5.5\% top-5 classification error compared to AlexNet 15.3\% top-5 classification error.

\subsubsection{Convolutional neural network}

This CNN model is more complicated and deeper in analyzation than all previous CNN models. Moreover, it uses "Inception" which concatenates filters of various sizes and dimensions into a single new filter. It contains two convolutions, two pooling and nine "Inception" layers. In this paper, three different RGB image sizes were used as an input $(64 \times 64$, $128 \times 128,256 \times 256$ ) corresponding to 3 different window sizes (section 3.3). By comparing them, we can decide which size gives the best results. Fig. 6 shows the illustration of CNN architecture used in this paper. 


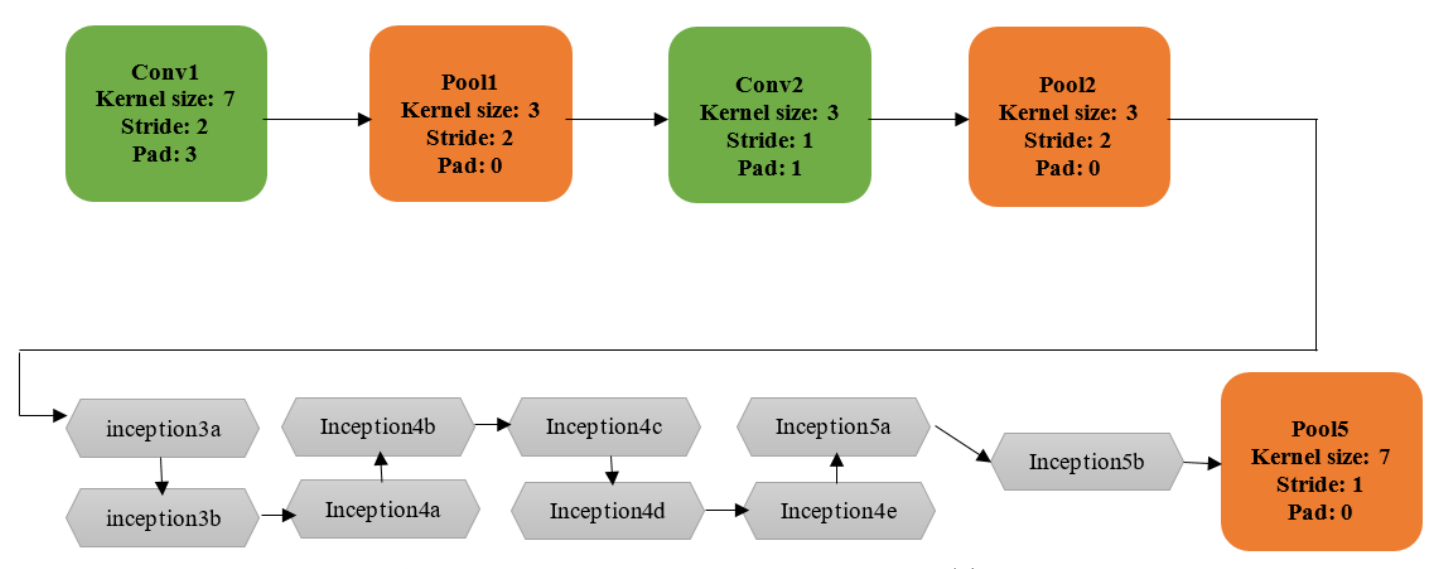

Figure 6. Illustration of GoogleNet's architecture

\subsubsection{CNN training process}

After the disease criticalness categorization in section 3.4, we have three small datasets for 3 different window sizes: each size includes 2,000 healthy radish images, 2,000 light Fusarium wilt, and 2,000 heavy Fusarium wilt. The entire dataset was randomly divided into training and validation set. The training and validation set occupied $75 \%$ and $25 \%$ of the entire dataset respectively, and the validation set was used for tuning the learning rate. In the training phase, the batch size was set to 90 and momentum was 0.9 . The learning rate was initially set to 0.01 and gradually reduced to 0.0001 according to the error rate of the validation set, as the parameter vector bounces around chaotically with a high learning rate, therefore it is ideal to step decay the learning rate. The training phase ran for 30 epochs, which took approximately 1 hour.

The system used for training CNN model was NVIDA DIGITS toolbox with Caffe framework. The experiments were implemented on a Linux machine, with Ubuntu 14.04, it used Intel® Core i7-5930K processor, four NVIDIA Titan X 12GB GPUs, four 3072 Cuda cores, and 64GB of DDR4 RAM.

\section{Experimental Results and Discussion}

\subsection{Evaluation Methodology}

\subsubsection{Classification evaluation protocol}

K-fold cross validation $(\mathrm{k}=3)$ is applied to evaluate the system performance. it divides the entire dataset into $\mathrm{k}$ roughly equal-sized disjoint subsets. Two subsets are used to train the proposed method, while the remaining subset is used to evaluate the performance of the method. This is repeated $\mathrm{k}$ times with differing choices of the remaining subset. For radish field 
classification, the confusion matrix is computed to assess the ability of our model to distinguish differing areas of radish fields (Radish, bare ground, and mulching film). The confusion matrix CM can be computed by:

$$
C M_{i j}=\sum_{R \in d} \mid\{r \in R \text { such that GroundTruth }(r)=i \text { and Prediction }(r)=j\}
$$

where $R$ is the ROIs and GroundTruth $(r)$ and Prediction $(r)$ denote the ground truth class label and predicted class label of an ROI $r$, respectively.

\subsubsection{Segmentation evaluation protocol}

The radish field segmentation using K-means clustering was accessed with different value of $\mathrm{K}(\mathrm{K}=3,5,10,15$, and 20$)$. Therefore, the results varied when different $\mathrm{K}$ were applied. In order to find the value of $\mathrm{K}$ which had the highest performance, the segmentation results were evaluated by calculating the pixel-level segmentation accuracy [20] (PSA). PSA is the most popular semantic segmentation measure which evaluates pixel-level classification accuracy. PSA is calculated in Equation (9)

$$
P S A=\frac{\sum_{i}^{n} C_{i i}}{\sum_{i}^{n} \sum_{j}^{n} C_{i j}}
$$

PSA was measured for different values of $K$ in $K$-means clustering $(K=3,5,10,15$, and 20), thus we can analyze the effect of the size of the clusters on segmentation performance.

\subsection{Radish Region Classification Results}

Table 1 and Table 2 show the confusion matrix of classification results on Dataset A (radish ROIs: 500, bare ground ROIs: 500, and mulching film ROIs: 500). After that, we calculated precision, recall and F-measure for SVM classifier and softmax classifier using the confusion matrix. The result is shown in Fig. 7; it is noticeable from the graph that softmax classifier achieves the overall performance over $90 \%$ whereas, for SVM the F-measure is $72 \%$ since correctly classified bare ground was only 342 per 500 samples. As the result, softmax classifier is chosen to be the default classifier in the model.

\begin{tabular}{|c|c|c|c|}
\hline Predicted & Radish & Bare Ground & Mulching Film \\
\hline Radish & 498 & 0 & 13 \\
\hline Bare ground & 1 & 491 & 104 \\
\hline Mulching film & 1 & 9 & 383 \\
\hline
\end{tabular}

Table 1. Confusion matrix for softmax classification 
Table 2. Confusion matrix for SVM classification

\begin{tabular}{|c|c|c|c|}
\hline Predicted & Radish & Bare Ground & Mulching Film \\
\hline Radish & 475 & 3 & 2 \\
\hline Bare ground & 18 & 342 & 6 \\
\hline Mulching film & 7 & 155 & 492 \\
\hline
\end{tabular}

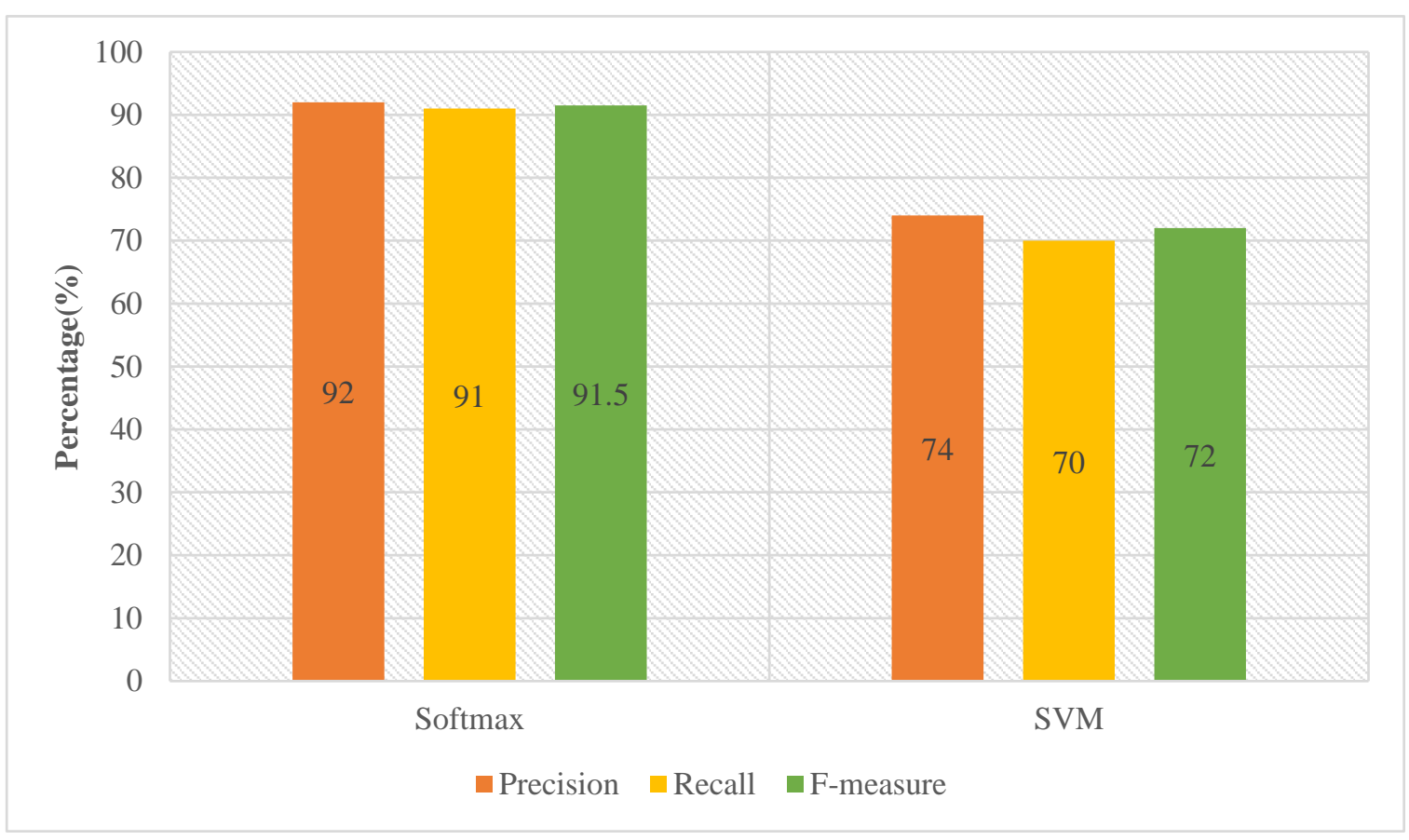

Figure 7. Comparison between SVM and softmax classifier on Dataset A

\subsection{Radish Field Segmentation Results}

Radish field segmentation accuracy was computed by pixel-level segmentation accuracy (PSA), it is also validated by 3-fold cross validation with same dataset and classifier used for radish field classification evaluation. 


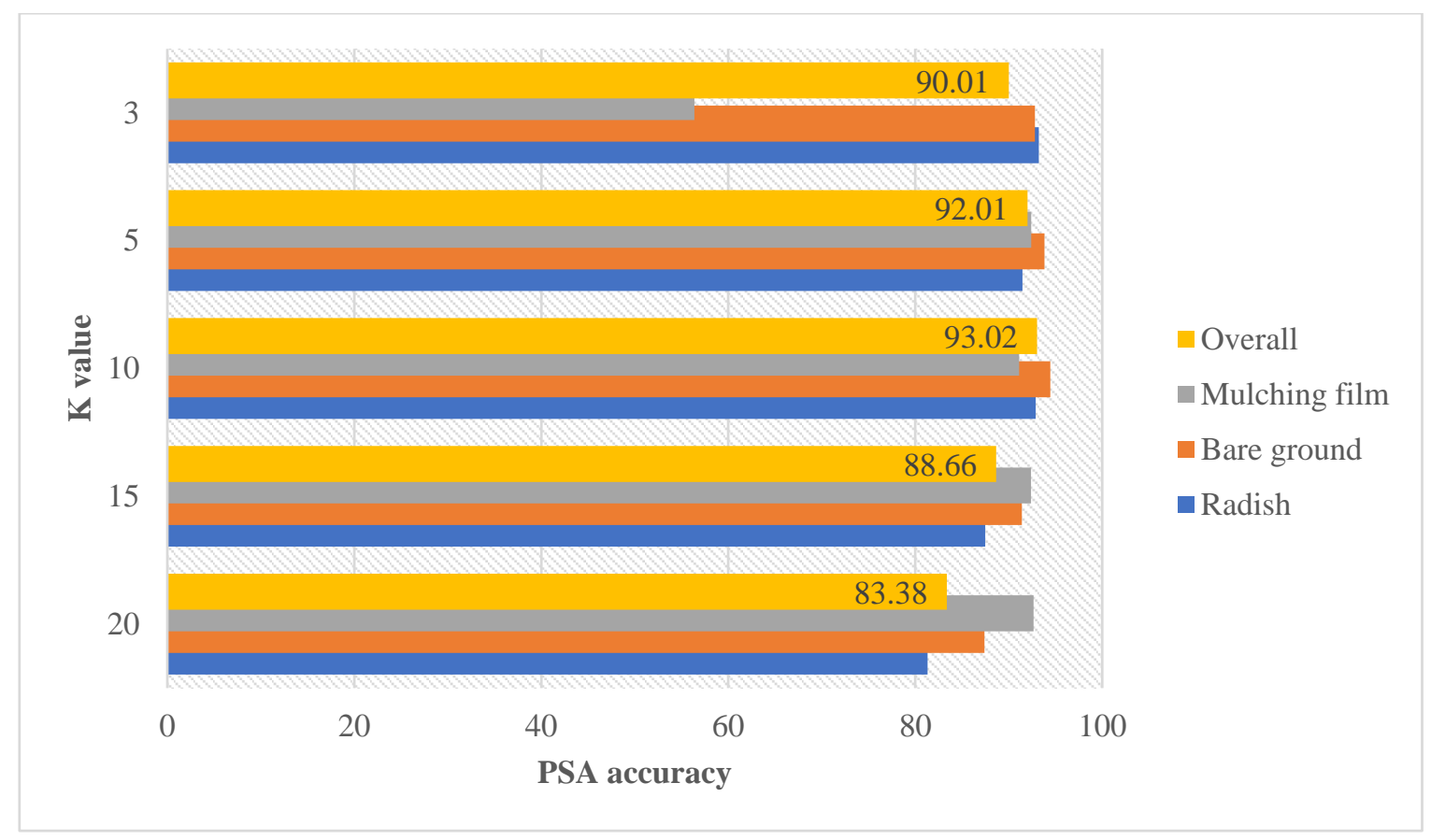

Figure 8. PSA evaluation on K-means with different K

Radish field segmentation results (PSA) with different cluster (K) values are presented in Fig. 8. As K increases, the overall PSA accuracy increases gradually to 93\%. However, when the value of $\mathrm{K}$ is greater than 10 , it gradually decreases. As the optimal value of $\mathrm{K}$ depends solely on the dataset, in case of this dataset, PSA was used to find the most suitable $\mathrm{K}$ value. With $\mathrm{K}=10$, the PSA accuracy was over $91 \%$ for radish, bare ground, and mulching film. The experimental results suggest that $\mathrm{K}=10$ is the optimal number of clusters for radish field segmentation.
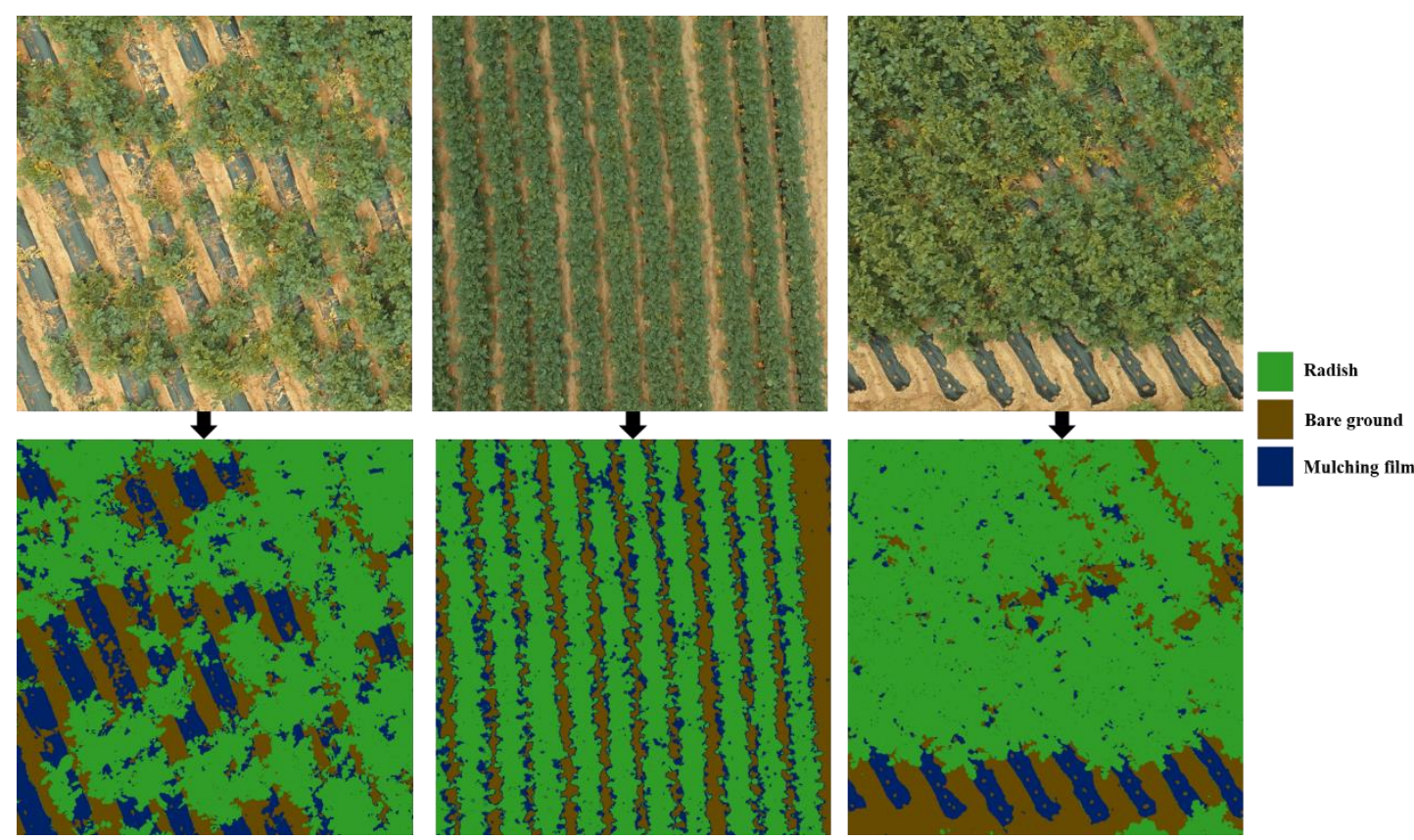

Mulching film

Figure 9. Radish field segmentation results 
Fig. 9 shows the segmentation results with $K=10$. The regions corresponding to radish, bare ground, and mulching film are correctly classified as labeled. Although, the segmentation result works great on separating radish, bare ground and mulching film, misclassified regions are also represented in Fig. 10. These include withered radishes that are mainly brown in color. Due to the similarity in color with bare ground, these regions were clustered together with bare ground by K-means clustering. The low performance is not caused by the radish field classifier but by the clustering method, as K-means clustering is based on three color channels as described in section 3.2
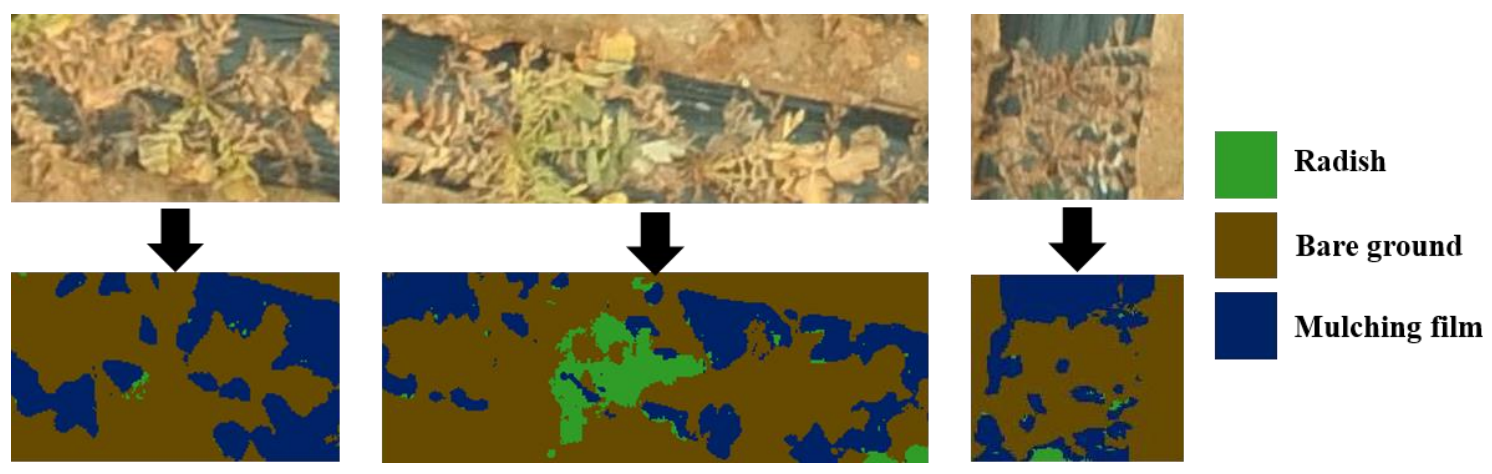

Figure 10. Wilt of radish segmentation results (Brown leaves was misclassified as bare ground)

\subsection{Fusarium Wilt of Radish Classification Results}

Fig. 11 shows the performance of Fusarium wilt of radish classification on different image sizes. Overall, the 128x128 image size dataset achieves the highest classification performance at over $90 \%$ for three classes. Taking a closer look into $128 \times 128$ window size, the model misclassifies two pairs of classes, the first pair is (normal, disease light), the second pair is (disease light, disease heavy), one possible reason is because the color value range threshold to distinguish between three classes was similar, therefore it was unable to assign the right class for some images. Besides the 128x128 size dataset, the results for $64 \times 64$ and 256x256 window sizes are not as good as $128 \times 128$ size dataset, thus in this study the $128 \times 128$ size dataset is deemed the optimal size. 

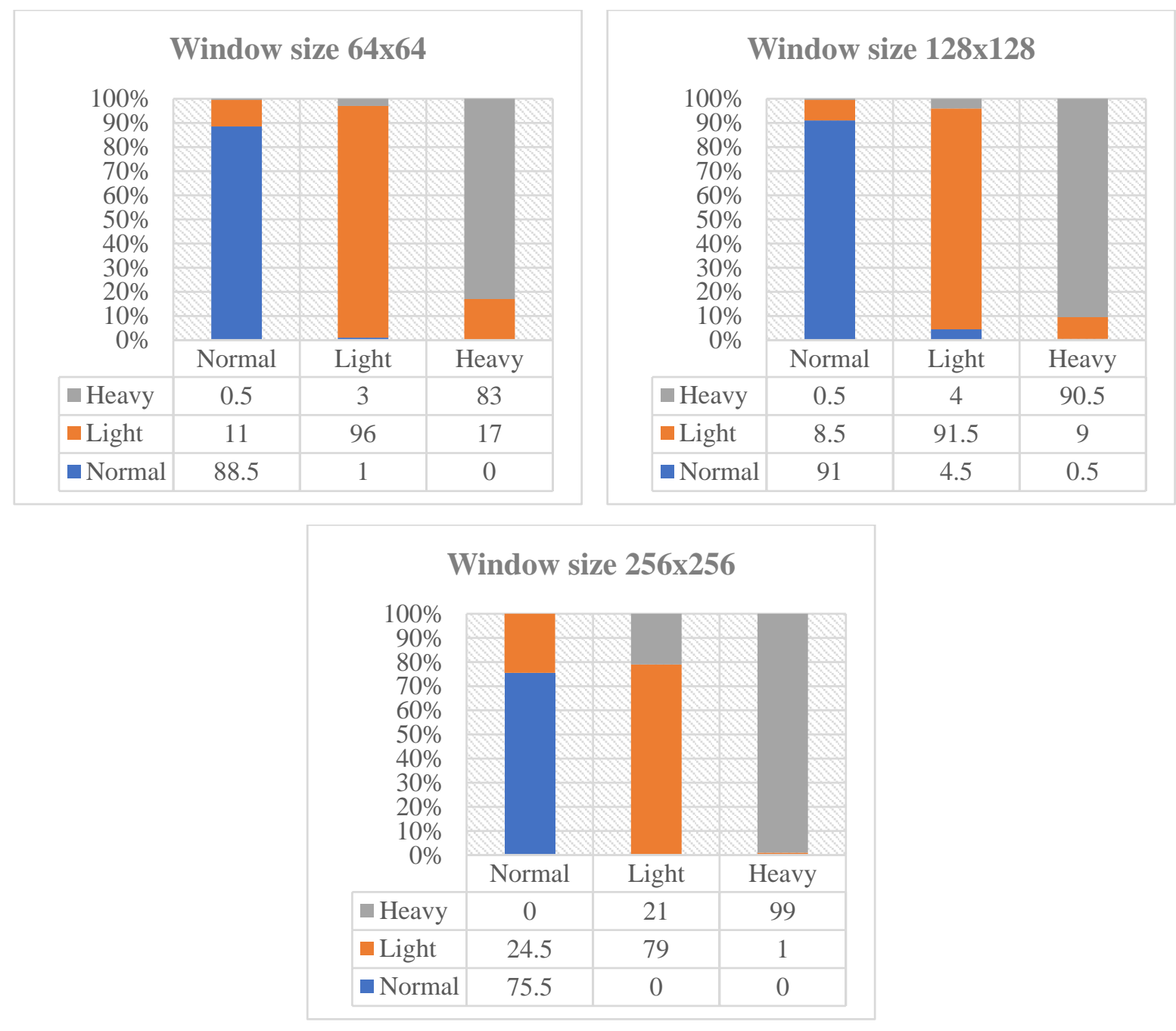

Figure 11. Fusarium wilt of radish classification results on different window sizes

Fig. 12 shows the Fusarium wilt of radish detection results on radish region images. Fig. 13 shows the detailed detection results of the Fusarium wilt of radish. Generally, the Fusarium wilt of radish grows from yellow to brown as the disease gets worse. As can be seen in Fig. 13, light-level Fusarium wilt of radishes (partly yellow) and heavy-level Fusarium wilt of radishes (mainly yellow) are successfully detected. However, some parts of the leaves are missing because browned radishes such as heavy-level Fusarium wilt of radish or dried radish are often segmented as bare ground in segmentation step they are discarded when only radish region images are extracted. This problem causes a reduction of Fusarium wilt of radish detection accuracy. 


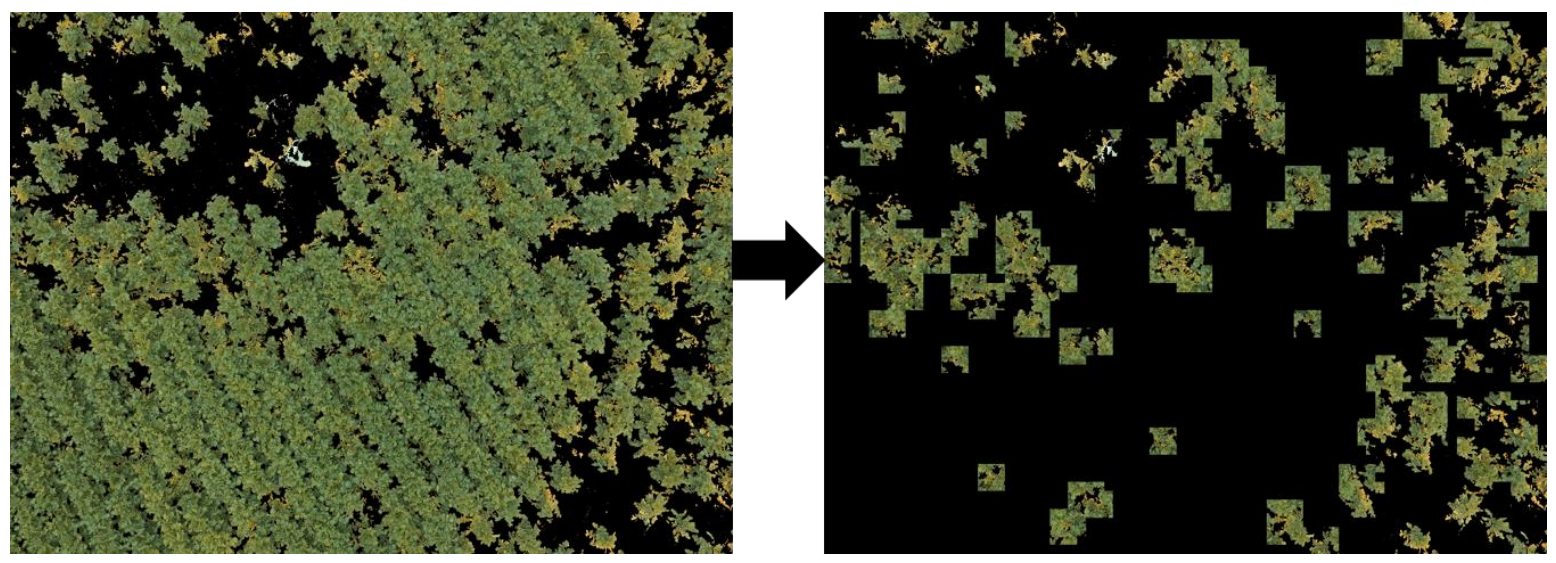

Figure 12. Extracted radish image (Left) and wilt of radish detection result in radish field (Right)

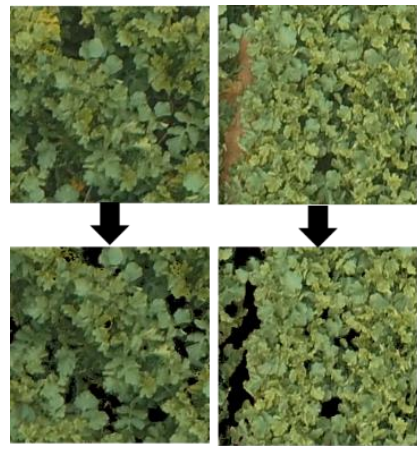

Healthy radish

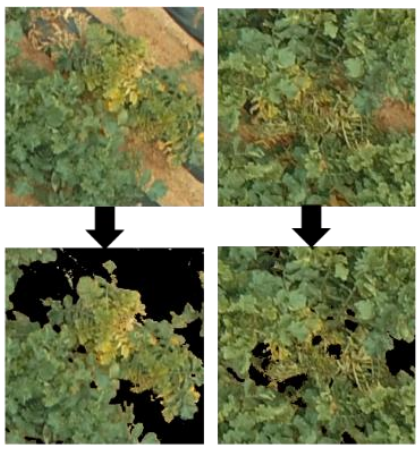

Disease light

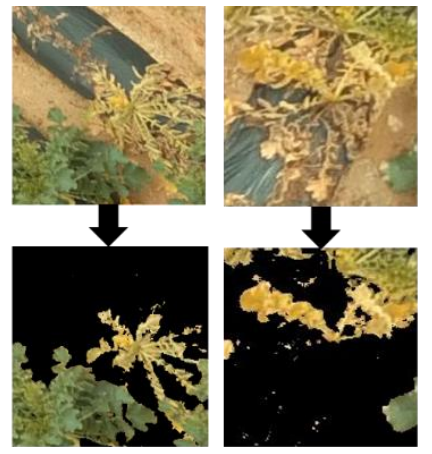

Disease heavy

Figure 13. Closed-range Fusarium wilt of radish detection

\section{Conclusions}

This study introduces an efficient framework to identify and detect different levels of Fusarium wilt on radish from healthy to disease heavy. Many techniques were implemented to improve the performance of the system such as extracted both color features and textures features from the image, segmented the field and select only radish region. Finally, GoogLeNet is able to detect Fusarium wilt of radish with the accuracy of over $90 \%$.

The model is capable of detecting Fusarium wilt of radish from UAV's images which has a great potential for reducing the labor cost in managing and preventing the disease, as well as ensuring radish sustainable production. The proposed system is able to detect Fusarium wilt on other type of crops including tomato, tobacco, banana because it is a common vegetable disease therefore the model plays an important role on maintaining the sustainability of crop yields.

In the future, several related issues will be study. Firstly, beside RGB image, infrared is often employed to monitor diseases and the infrared filter is easy to equip on existing UAV's camera lens. A methodology that combines RGB images and infrared images will probably improve the performance of the model. Secondly, several pre-processing techniques and deep learning models to improve the performance of this problem will be studied. 


\section{Acknowledgments}

This work was supported by Korea Institute of Planning and Evaluation for Technology in Food, Agriculture, Forestry and Fisheries(IPET) through Agri-Bio Industry Technology Development Program, funded by Ministry of Agriculture, Food and Rural Affairs(MAFRA) (316033-04-2-338 SB030)

\section{References}

1. Mace, M., Fungal wilt diseases of plants. 2012: Elsevier.

2. Candiago, S., et al., Evaluating multispectral images and vegetation indices for precision farming applications from UAV images. Remote Sensing, 2015. 7(4): p. 4026-4047.

3. Tang, L. and G. Shao, Drone remote sensing for forestry research and practices. Journal of Forestry Research, 2015. 26(4): p. 791-797.

4. Matese, A., et al., Intercomparison of UAV, aircraft and satellite remote sensing platforms for precision viticulture. Remote Sensing, 2015. 7(3): p. 2971-2990.

5. Khan, M.A., et al., UAV-Based Traffic Analysis: A Universal Guiding Framework Based on Literature Survey. Transportation Research Procedia, 2017. 22: p. 541-550.

6. Yuan, C., Y. Zhang, and Z. Liu, A survey on technologies for automatic forest fire monitoring, detection, and fighting using unmanned aerial vehicles and remote sensing techniques. Canadian journal of forest research, 2015. 45(7): p. 783-792.

7. Qi, J., et al., Search and Rescue Rotary-Wing UAV and Its Application to the Lushan Ms 7.0 Earthquake. Journal of Field Robotics, 2016. 33(3): p. 290-321.

8. Ren, D.D., S. Tripathi, and L.K. Li, Low-cost multispectral imaging for remote sensing of lettuce health. Journal of Applied Remote Sensing, 2017. 11(1): p. 016006-016006.

9. Ahmad, J., et al., Saliency-weighted graphs for efficient visual content description and their applications in real-time image retrieval systems. Journal of Real-Time Image Processing, 2015: p. 1-17.

10. Ahmad, J., et al., Multi-scale local structure patterns histogram for describing visual contents in social image retrieval systems. Multimedia Tools and Applications, 2016. 75(20): p. 12669-12692.

11. Lowe, D.G., Distinctive image features from scale-invariant key points. International journal of computer vision, 2004. 60(2): p. 91-110.

12. Wang, C., et al. Spatial weighting for bag-of-features based image retrieval. in International Symposium on Integrated Uncertainty in Knowledge Modelling and Decision Making. 2013. Springer.

13. Krizhevsky, A., I. Sutskever, and G.E. Hinton. Imagenet classification with deep convolutional neural networks. in Advances in neural information processing systems. 2012.

14. Szegedy, C., et al. Going deeper with convolutions. in Proceedings of the IEEE conference on computer vision and pattern recognition. 2015.

15. Lin, M., Q. Chen, and S. Yan, Network in network. ICLR, 2014.

16. Ojala, T., M. Pietikainen, and T. Maenpaa, Multiresolution gray-scale and rotation invariant texture classification with local binary patterns. IEEE Transactions on pattern analysis and machine intelligence, 2002. 24(7): p. 971-987. 
17. Vincent, P., et al., Stacked denoising autoencoders: Learning useful representations in a deep network with a local denoising criterion. Journal of Machine Learning Research, 2010. 11(Dec): p. 3371-3408.

18. Simonyan, K. and A. Zisserman, Very deep convolutional networks for large-scale image recognition. CoRR, abs/1409.1556, 2014.

19. Russakovsky, O., et al., Imagenet large scale visual recognition challenge. International Journal of Computer Vision, 2015. 115(3): p. 211-252.

20. Csurka, G., et al. What is a good evaluation measure for semantic segmentation? in BMVC. 2013.

21. $\mathrm{Li}, \mathrm{Li}$, and Ken Choi. "Activity-driven optimised bus-specific-clock-gating for ultra-low-power smart space applications." IET communications 5.17 (2011): 2501-2508.

22. Dang, Minh, and Duc Duong. "Improvement methods for stock market prediction using financial news articles." Information and Computer Science (NICS), 2016 3rd National Foundation for Science and Technology Development Conference on. IEEE, 2016.

23. Chang, Chih-Chung, and Chih-Jen Lin. "LIBSVM: a library for support vector machines." ACM transactions on intelligent systems and technology (TIST) 2.3 (2011): 27.

24. Kieu, Tung, et al. "Mining top-k co-occurrence items with sequential pattern." Expert Systems with Applications 85 (2017): 123-133.

25. Cui, Zhihua, and Xiaozhi Gao. "Theory and applications of swarm intelligence." (2012): 205-206.

26. Udoyara Sunday Tim, and Sugam Sharma. Harnessing the Power of Big Data and Big Data Analytics in Agriculture: Opportunities and Challanges. 2014.

27. Chen, Chen, et al. "Latency estimation based on traffic density for video streaming in the internet of vehicles." Computer Communications 111 (2017): 176-186.

28. Qiu, Tie, et al. "A Robust Time Synchronization Scheme for Industrial Internet of Things." IEEE Transactions on Industrial Informatics (2017).

29. Samuel, Oluwarotimi Williams, et al. "An integrated decision support system based on ANN and Fuzzy_AHP for heart failure risk prediction." Expert Systems with Applications 68 (2017): 163-172.

30. Liao, Xin, et al. "Medical JPEG image steganography based on preserving inter-block dependencies." Computers \& Electrical Engineering (2017).

31. Zhang, Ruijie, et al. "Medical image classification based on multi-scale non-negative sparse coding." Artificial Intelligence in Medicine (2017).

32. Liang, Wei, et al. "SIRSE: A secure identity recognition scheme based on electroencephalogram data with multi-factor feature." Computers \& Electrical Engineering (2017).

33. Samuel, Oluwarotimi Williams, et al. "Pattern recognition of electromyography signals based on novel time domain features for amputees' limb motion classification." Computers \& Electrical Engineering (2017). 